\title{
The treatment of allergic rhinitis in asthmatic children and adolescents: practical outcomes from the real-world "ControL'Asma" study
}

\author{
${ }^{1}$ Allergy Center, Istituto Giannina Gaslini, Genoa, Italy \\ ${ }^{2}$ Pediatrics Clinic, Pediatrics Department, Policlinico San Matteo, University of Pavia, Pavia, Italy \\ ${ }^{3}$ Allergy Clinic, Casa di Cura Villa Montallegro, Genoa, Italy \\ *E. Anastasio, I. Brambilla, C. Caffarelli, L. Chini, R. Ciprandi, V. De Vittori, M. Duse, M. E. Di Cicco, L. Indinnimeo, A. Kan- \\ tar, M. Leone, A. Licari, G. Marinelli, V. Moschese, R. Olcese, D. G. Peroni, A. Pistorio, C. Salmaso, M. Silvestri, A. M. Zicari
}

\section{KEY WORDS}

Allergic rhinoconjunctivitis; asthma; control; children.

\author{
Corresponding author \\ Giorgio Ciprandi \\ Allergy Clinic \\ Casa di Cura Villa Montallegro \\ via P. Boselli 5 \\ 16146 Genoa, Italy \\ E-mail: gio.cip@libero.it
}

\section{Doi}

10.23822/EurAnnACI.1764-1489.171

To the Editor,

Allergic rhinitis (AR) affects up to $40 \%$ of children and adolescents (1). AR is characterized by a type 2 inflammation, including allergen-specific $\mathrm{IgE}$ production, eosinophilic infiltrate, and T helper 2(Th2)-derived cytokines (2). T regulatory cells' specific and functional defect promotes the typical Th2 polarization in allergic patients (2). Asthma is the most common chronic disease of childhood and adolescence (3). Asthma management is, therefore, a daily challenge in pediatric practice (4). Asthma is a heterogeneous disease, usually characterized by chronic airway inflammation (3). To define clinical, functional, and immunopathological patterns allows identifying asthma phenotypes and endotypes (5). In this regard, the allergic asthma phenotype is the most common in childhood and is defined when asthma symptoms and airway eosinophilic inflammation are associated with inhalation of the sensitizing allergen (3). There is also a close link between eosinophilic airway inflammation and airflow limitation (6). Therefore,
$\mathrm{AR}$ and asthma share common pathogenic mechanisms and are frequently associated (7). In clinical practice, the concomitant treatment of asthma and AR can commonly produce practical problems. The relief of symptoms and control of airway inflammation represents the cornerstone of their management, even though some exceptions $(3,8)$. Symptoms relief need bronchodilator use in asthma and essentially antihistamines (anti-H1) in AR, but inflammation resolution depends on inhaled corticosteroids (ICS) in asthma and intranasal corticosteroids in AR. However, the overtreatment of both diseases may generate adverse events, mainly concerning corticosteroids that may induce relevant issues (9). Allergen immunotherapy (AIT) could represent the shared treatment committed to restoring allergen tolerance, revert $\mathrm{Th} 2$ polarization, and ultimately dampen type 2 inflammation (10).

The Italian Society of Pediatric Allergy and Immunology recently established a prospective study ("ControL'Asma") to investigate the asthma control in children and adolescents managed in clini- 
cal practice. This research has been paid attention to the concomitant treatment of AR in children and adolescents with asthma. This cross-sectional study included a series of asthmatic children and adolescents consecutively visited across 10 Italian Pediatric Allergy centers. The centers are in Genoa, Bergamo, Milan, Pavia, Parma, Pisa, Rome (3 centers), and Catanzaro. All patients were currently treated according to the GINA guidelines based on the asthma control level and AR guidelines (11). The visit included careful history, mainly concerning asthma duration, current use of asthma and AR medications, including inhaled corticosteroids dosage (ICS) expressed as beclomethasone equivalence, oral corticosteroids use, rhinitis and allergy comorbidity, clinical examination, lung function testing (including bronchodilation testing), asthma control level according to the GINA guidelines (3). The Ethics Committee initially approved the procedure of the Istituto Giannina Gaslini of Genoa (code number: 22253/2017; in the Italian Project "ControL'Asma” promoted by the Italian Society of Pediatric Allergy and Immunology). All the other Review Ethics Committees further approved the study procedure, and written informed consent was obtained from all parents. Clinical data were recorded by an electronic case report form designed express- ly for this study. Descriptive statistics of the study patients were firstly calculated; qualitative data were reported in terms of absolute frequencies and percentages; quantitative data were reported in terms of medians, first and third quartiles $\left(1^{\text {st }}-3^{\text {rd }} Q\right)$.

The normality of distributions was evaluated using the Shapiro-Wilk test.

The statistical software "Statistica" (version 9, StatSoft Corporation, Tulsa, OK, USA) was used for all the analysis, and the software "Stata" (version 11, Stata Corporation, College Station, TX, USA) was used to calculate the Shapiro-Wilk.

Globally, 480 subjects were enrolled; 423 (88.1\%) had AR comorbidity. Table I reports the clinical characteristics of the patients with both diseases. Signally, there was a male predominance $(70 \%)$; well-controlled asthma was 55\%, moderate-severe AR in $14 \%$. ICS were used in $75 \%$ and OCS in $23 \%$, and antileukotrienes in $24 \%$. Antihistamines were used in $57.6 \%$, intranasal corticosteroids in 53\%, and both combined in 35. Notably, about $3 / 4$ of patients used pharmacotherapy for AR, only $19.5 \%$, in contrast, used AIT. As regards sensitization, house dust mites were the most common sensitizing allergen (78\%), followed by grasses $(60 \%)$, olive tree $(36 \%)$, cat $(31 \%)$, hazelnut tree $(25 \%)$,

Table I - Description of the study patients with allergic asthma and rhinitis.

\begin{tabular}{|c|c|c|c|}
\hline Clinical characteristics & & Sensitization to & \\
\hline Age (years) & $11.4(9.4-13.8)^{*}$ & House dust mites & $78.0 \%$ \\
\hline Males & $69.3 \%$ & Grasses & $59.7 \%$ \\
\hline Well-controlled asthma & $54.9 \%$ & Cat & $30.6 \%$ \\
\hline Partly controlled asthma & $32.5 \%$ & Hazelnut tree & $24.7 \%$ \\
\hline Mild intermittent AR & $37.9 \%$ & Dog & $20.7 \%$ \\
\hline Moderate/severe intermittent AR & $9.2 \%$ & Alternaria & $19.6 \%$ \\
\hline Mild persistent AR & $47.6 \%$ & Cypress & $16.4 \%$ \\
\hline Moderate/severe persistent AR & $5.2 \%$ & Parietaria & $16.3 \%$ \\
\hline OCS: at least 1 course/year & $22.7 \%$ & & \\
\hline LABA & $35.5 \%$ & & \\
\hline Anti-LTC & $24.3 \%$ & & \\
\hline Intranasal corticosteroids & $53.0 \%$ & & \\
\hline Anti- $\mathrm{H}_{1}$ & $57.6 \%$ & & \\
\hline Intranasal corticosteroids + Anti- $\mathrm{H}_{1}$ & $35.0 \%$ & & \\
\hline Allergen-specific Immunotherapy & $19.5 \%$ & & \\
\hline
\end{tabular}

${ }^{*}$ Median values and $1^{\text {st }}$ and $3^{\text {rd }}$ quartiles. 
birch (23.4\%), dog (21\%), Alternaria (20\%), cypress (16\%), Parietaria (16\%), and Compositae (15\%). There were geographical differences concerning the distribution of specific sensitizations consistently with previous studies (12-14). Nevertheless, there was no significant difference in terms of treatments and disease severity among the centers. It mainly depended on the uniform sharing to International guidelines for asthma and rhinitis and the fact that all centers were third-level pediatric allergy clinics. The current study demonstrated that AR is prevalent comorbidity in children and adolescents with asthma as affected by almost $90 \%$ of the whole sample. This outcome underlined the clinical relevance of the concept of united airways disease (15). However, corticosteroids were the most common medication as ICS was used in nearly all subjects and intranasal corticosteroids in more than half. However, antihistamines (mostly oral) were the first-choice treatment for AR. These findings arouse some concern concerning potential adverse events related to medication use. On the other hand, AIT was used only in $20 \%$ of patients. It is well known that medications do not cure the allergy, as symptoms and inflammation quickly recur after their suspension $(16,17)$. AIT should represent the choice treatment of AR and allergic asthma as restores immunological and clinical tolerance toward the causal allergen, may prevent allergy worsening, and its effects are longlasting over time. Also, there is a predominance of sensitization to perennial allergens, such as it means that allergic inflammation persists throughout the year. Anti-inflammatory medications should be used for a long time, with the problem of side effects. As a result, a more rational approach should be pursued in asthmatic children and adolescents.

The current study had some limitations, mainly concerning the cross-sectional design and the lack of biomarkers assessment. However, a follow-up study is ongoing. Moreover, the strength of this study was the nationwide size that provides generalizability of the outcomes. This real-world study may also provide information more adherent to the daily practice that studies involving selected patient populations that rarely mirror the real situation (18).

In conclusion, the present study demonstrated that AR was common asthma comorbidity in children and adolescents. Well-controlled asthma affected only half of the patients despite the use of corticosteroids was widespread and perennial allergy was also predominant. AIT was scarcely prescribed. These outcomes have to convince that more efforts should be made to improve asthma management in children and adolescents.

\section{Contributors}

MAT designed the study, collected patients, and discussed the paper, GLM discussed and revised the paper, GC wrote the paper.

\section{Conflict of interests}

The authors declare that they have no conflict of interests.

\section{References}

1. Ebert CS Jr, Pillsbury HC 3rd. Epidemiology of allergy. Otolaryngol Clin North Am 2011;44:537-48.

2. Palomares O, Akdis M, Martín-Fontecha M, Akdis CA. Mechanisms of immune regulation in allergic diseases: the role of regulatory T and B cells. Immunol Rev 2017;278:219-36.

3. Cosmi L, Liotta F, Maggi L, Annunziato F. Role of Type 2 Innate Lymphoid Cells in Allergic Diseases. Curr Allergy Asthma Rep 2017;17(10):66.

4. Global Initiative for Asthma. Global strategy for asthma management and prevention, 2019. Available at: www.ginasthma.org. Last access date: 27.11.2020.

5. Chipps BE, Bacharier LB, Farrar JR, et al. The pediatric asthma yardstick. Ann Allergy Asthma Immunol 2018;120:559-79.

6. Ghibril N, Casale T, Custovic A, Phipatanakul W. Allergic Endotypes and Phenotypes of Asthma. J Allergy Clin Immunol Pract 2020;8:429-40.

7. Ciprandi G, Tosca MA, Marseglia GL, Klersy C. Relationships between allergic inflammation and nasal airflow in children with seasonal allergic rhinitis. Annals Allergy Asthma Immunol 2005;94:258-61.

8. Passalacqua G, Ciprandi G. Pasquali M, Guerra L, Canonica GW. An update on the asthma-rhinitis link. Curr Opin Allergy Clin Immunol 2004;4:177-83.

9. Wise SK, Lin SY, Toskala E, et al. International Consensus Statement on Allergy and Rhinology: Allergic Rhinitis. Int Forum Allergy Rhinol 2018;8(2):108-352.

10. Ahmed H, Turner S. Severe Asthma in Children-A Review of Definitions, Epidemiology, and Treatment Options in 2019. Pediatr Pulmonol 2019;54:778-87.

11. Pfaar O, Agache I, de Blay F, et al. Perspectives in Allergen Immunotherapy: 2019 and Beyond. Allergy 2019;74(Suppl. 108):3-25.

12. Ciprandi G, Comite P, Mussap M, et al. Profiles of birch sensitization (Bet v 1, Bet v 2, Bet v 4) and oral allergy syndrome across Italy. J Inv All Clin Immun 2016;26:244-8.

13. Ariano R, Cecchi L, Voltolini S, Quercia O, Scopano E, Ciprandi G. Parietaria pollination duration: myth or fact? Eur Annals Allergy Clin Immunol 2017;49:6-10.

14. Ciprandi G, Scala E, Ariano R. Phleum pratense molecular pattern across Italy. Eur Annals Allergy Clin Immunol 2017;49:176-80.

15. Bousquet J, Schunemann HJ, Togias A, et al. Next-generation Allergic Rhinitis and Its Impact on Asthma (ARIA) Guidelines for Allergic Rhinitis Based on Grading of Recommendations Assessment, Development and Evaluation (GRADE) and Real-World Evidence. J Allergy Clin Immunol 2020;145:70-80.e3.

16. Yii ACA, Tay T-R, Choo XN, Koh MSY, Tee AKH, Wang D-Y. Precision Medicine in United Airways Disease: A "Treatable Traits" Approach. Allergy 2018;73:1964-78.

17. Chang DV, Teper A, Balinotti J, Castro Simonelli C, Garcia-Bournissen F, Kofman C. Exhaled Nitric Oxide Predicts Loss of Asthma Control in Children After Inhaled Corticosteroids Withdrawal. Pediatr Pulmonol 2019;54:537-43.

18. Ciprandi G, Ricca V, Ferrero P, et al. Duration of anti-inflammatory and symptomatic effects after suspension of intranasal corticosteroid in persistent allergic rhinitis. Eur Ann Allergy Clin Immunol 2004;36:63-6. 